\title{
Association of renal cortical echogenicity and renal function in chronic kidney disease - sonographic study
}

Kodikara I ${ }^{1}$, Gamage DTK ${ }^{2}$, Nilmini KWGP ${ }^{1}$, Nanayakkara BG ${ }^{1}$, Ilayperuma I ${ }^{1}$

${ }^{\prime}$ Department of Anatomy, Faculty of Medicine, University of Ruhuna, Galle.

${ }^{2}$ Base Hospital Tissamaharama, Tissamaharama.

\section{Introduction}

Ultrasonographically measured renal cortical echogenicity (RCE)is known to increase in chronic kidney disease (CKD) andof value in predicting the degree of renal function impairment. Renal function is routinely assessed by serum creatinine levels (Scr) and estimated glomerular filtration rate (eGFR). The aim of the study was to evaluate the association of RCE with Scr and eGFR in CKD subjects.

\section{Methodology}

Diagnosed CKD patients $(\mathrm{n}=100)$ were recruited after excluding the subjects with history of renal surgeries, acute renal failure or known liver disease. $\mathrm{SCr}$ values done within the last three months were recorded. eGFR was calculated using a standard formula. RCE was evaluated by a single Radiologist with linear probe. RCE was graded as grade 1,2 and 3 in comparison to the liver echogenicity (for right kidney) and splenic echogenicity (for the left kidney) according to the previous studies.

\section{Results}

The study population; $70 \%$ males and $30 \%$ females, aged $50-89$ years (mean $68 \pm 8.4$ years). Mean $\mathrm{SCr}$ was $1.88 \pm 0.60$ $\mathrm{mg} / \mathrm{dL}$ and eGFR was $43.3 \pm 11.85$ $\mathrm{ml} / \mathrm{min} / 1.73 \mathrm{~m}^{2}$. RCE grade 1 (23\%), grade $2(55 \%)$ and grade $3(22 \%)$ were identified in the study population. RCE demonstrated a significant negative correlation with eGFR ( $\mathrm{r}-0.46, \mathrm{p}<0.01$ ) and significant positive correlation with $\operatorname{Scr}(\mathrm{r}-0.46, \mathrm{p}<0.01)$. However, RCE was not affected by the age of the subjects ( $\mathrm{r}-$ $0.09, \mathrm{p}=0.18$ ).

\section{Discussion and Conclusions}

According to the study results RCE appears to be of value in predicting renal function in CKD. Further studies with larger sample sizes are recommended to strengthen the findings. 